\title{
Universal propulsion harnessing the global anisotropy of the physical space
}

\author{
Yuriy Alexeevich Baurov 1, 4, *, Lorenzo Albanese², Francesco Meneguzzo², \\ Valeriy Aleksandrovich Menshikov ${ }^{3}$ \\ ${ }^{1}$ Closed Joint Stock Company Research Institute of Cosmic Physics, 141070, Moscow Region, Korolyov, Pionerskaya, 4, Russia \\ ${ }^{2}$ National Research Council - Institute of Biometeorology, I-50145 Firenze, Via Caproni, 8, Italy \\ ${ }^{3}$ Non-commercial partnership (NP) "The International Committee of the Project of the International Global Aerospace Monitoring and \\ Forecasting” (IGMASS), 141092, Moscow region, Yubileynyy, Lesnaya, 14, Office 305, Russia \\ ${ }^{4}$ Present address: Hotwater Srl, I-56024 San Miniato (PI), Via Gioberti, 15, Italy
}

\section{Email address:}

baurov@mail.ru (Y. A. Baurov), 1.albanese@ibimet.cnr.it (L. Albanese), f.meneguzzo@ibimet.cnr.it (F. Meneguzzo), vamenshikov@mail.ru (V.A. Menshikov)

\section{To cite this article:}

Yuriy Alexeevich Baurov, Lorenzo Albanese, Francesco Meneguzzo, Valeriy Aleksandrovich Menshikov. Universal Propulsion Harnessing the Global Anisotropy of the Physical Space. American Journal of Modern Physics. Vol. 2, No. 6, 2013 , pp. 383-391. doi: 10.11648/j.ajmp.20130206.26

\begin{abstract}
A simple electro-mechanical device is shown to harness the global anisotropy of the physical space in order to generate a traction force having an universal character because it doesn't involve the exchange of momentum either with any contact surface or conventional medium such as water or air, or any ordinary ejected substance such as exhaust gases or ionized particles, but with the physical space meant as a physical object filled generally with "cold" and locally with "warm" dark matter. The new force of nature investigated in this article is predicted by the non-gauge cosmological physical theory of "byuon". This theory is based upon very few basic axioms, among which the marginal global anisotropy of the physical space caused by the existence of a cosmological vector potential $\mathrm{A}_{\mathrm{g}}$ having coordinates in the second equatorial coordinate system as follows: right ascension $\alpha=300^{\circ} \pm 10^{\circ}$, declination $\delta=36^{\circ} \pm 10^{\circ}$. The new predicted force was experimentally studied during about 30 years as well as was revealed as the primary cause of changes in the decay rate of radioactive elements and for a wide variety of astrophysical and geophysical phenomena. The new force is as well predicted to be generally anisotropic, repulsive, non-local and non-linear; its existence is further proved in this article as well as its exploitability to move objects in any medium is demonstrated by means of few prototypes of the new concept thruster. Results collected during 2012-2013 do suggest that values of specific power as low as $330 \mathrm{~W} / \mathrm{N}$ can be practical, i.e. about 40 times smaller than the best corresponding figures of electric propulsion thrusters used to move satellites and spacecrafts, suggesting the possibility of important developments for the propulsion of space vehicles.
\end{abstract}

Keywords: Global Anisotropy, Theory of Byuon, Traction Force, Propulsion, Space Flights

\section{Introduction}

The history of the detection of the global anisotropy of the physical space as well as its attribution to a cosmological vector potential in the frame of the non-gauge theory of byuon was recently summarized and discussed in this journal [1].

In the earlier 1990s' the basic experiments were performed allowing the early detection of a fundamental anisotropy of the physical space as well as of a new non-gauge interaction, different from the four known ones (strong, weak, electromagnetic, and gravitational)[2,3]. Besides the lack of gauge invariance, an important feature of this new interaction is just its anisotropy that arises in a wide range of sizes, from the weak interactions, i.e. $10^{-19}$ $\mathrm{m}[4]$, up to the size of our Galaxy, i.e. $10^{21} \mathrm{~m}[5]$, and more[6].

The physical nature of the new force is explained by the theory of byuon, a non-gauge theory of the formation of the physical space and the world of elementary particles from some unobservable objects called "byuons" [7-9].

The proposed new force of nature arising basically from the local minimization of an energy-weighted sum of the potentials of all known physical force fields [10], and the momentum exchange occurring with the physical space "filled" with dark matter [9], suggests that, if harnessed in 
the form of a traction force, it could be used for universal propulsion because independent of any surrounding medium as well as of any substance to be ejected.

This idea requires at least the following two steps before it could possibly be implemented into useful thrusters:

- Detection of the proposed new force of nature in the form of traction force by means of simple devices and repeatable experiments;

- Demonstration of the competitiveness of the mentioned universal propulsion at least in terms of energy efficiency and at least in comparison to the most energy-intensive thrusters, i.e. space thrusters.

Electric Propulsion (EP) devices and especially Hall Effect Thrusters (HETs), based upon the ionization of an working gas, usually Xenon, and the acceleration of the resulting plasma by means of electromagnetic fields are currently largely used onboard geostationary satellites for orbit maintenance and de-orbiting at the end of their lifetime, competing with usual chemical propulsion engines mainly thanks to the larger ion ejection velocity, in turn leading to a consistent saving of the propellant mass by 5 to 10 times [11], as well as are deemed the most promising for both unmanned and manned missions to Mars [11,12].

The best figures for the thrust supplied by the electric thrusters so far operated on space flight vehicles, i.e. HETs and Ion Thrusters (ITs), are included in the range $10^{-3} \mathrm{~N}$ to $0.4 \mathrm{~N}$ corresponding to electric power between $300 \mathrm{~W}$ and $5000 \mathrm{~W}$, which means a specific power not lower than $12500 \mathrm{~W} / \mathrm{N}$, a figure typical of HETs [13]. The last figure of specific power will be used in this article as a benchmark in the field of space propulsion.

The findings shown and discussed in this paper represent substantial and original advancements over a previous article [14] which will be sometimes referred to in the following.

The paper is organized as follows: Section 2 describes the fundamental physical theory, developed on the basis of the global anisotropy of the physical space, from which the new force of nature arises (Section 2.1.), and the basics of the new principle of universal propulsion as well as earlier experiments (Section 2.2.). In Section 3, the experimental set-up and methods are presented. In Section 4, the original results obtained with the most recent experimental runs carried out during November, 2012 till September, 2013 are shown and discussed. The main conclusions and targets for future research are presented in Section 5.

\section{Theoretical Insights}

\subsection{The Global Anisotropy of the Physical Space and the Theory of Byuon}

As mentioned in Section 1., the detection of the global anisotropy of the physical space connected with a cosmological vector potential as a new universal constant ofnature, in the frame of the non-gauge theory of byuon, was recently discussed in this journal [1]; therefore, only a short overview will be provided below.

According to the theory of byuon a fraction of the mass of the elementary particles, associated with the formation of their inner physical space and up to the value $\Delta \mathrm{m} \cdot \mathrm{c}^{2}=33$ $\mathrm{eV}$ in value, is proportional to the modulus of a summary potential $l \mathrm{~A}_{\Sigma}$, i.e. the sum of the potentials of all known force fields calculated using the energy relation expressed by Eq. (3.1.) in[10]. Such summary potential cannot exceed, by magnitude, the modulus of the cosmological vectorial potential $\mathrm{A}_{\mathrm{g}}$, a new fundamental constant having absolute value $\left|\mathrm{A}_{\mathrm{g}}\right| \approx 1.9 \cdot 10^{5} \mathrm{~T} \cdot \mathrm{m}$.

As the result of the action of the field potentials (decreasing $\left|\mathrm{A}_{\Sigma}\right|$ ), each particle gains an energy $\Delta \mathrm{m} \cdot \mathrm{c}^{2}$, c being the speed of light, that corresponds to a new force of nature throwing substance out of the region with the weakened $A_{\Sigma}$. Experimental investigations with the use of gravimeters and magnets and plasma systems[15], as well as the measurements of changes in the $\beta$-decay rate of radioactive elements[4,16], that were later confirmed by independent researches[17], have shown that the substance is ejected from the region with the weakened $A_{\Sigma}$ along a cone with an angular opening about $100^{\circ}$ around the vector determining the global anisotropy of the physical space and having the following astronomical coordinates in the second equatorial system: $\alpha \approx 293^{\circ} \pm 10^{\circ}, \delta \approx 36^{\circ} \pm 10^{\circ}$ where $\alpha$ is the right ascension and $\delta$ is the declination, the former value being later updated to the following: $\alpha=300^{\circ} \pm 10^{\circ}[1]$.

Fig. 1 shows the direction of the cosmological vector potential $A_{g}$ projected onto the Earth's orbital plane, representing the direction of the global anisotropy of the physical space.

The analysis of a long run of experiments has shown that the new force has a nonlinear and nonlocal character and can be represented as a complex series in terms of changes of the summary potential $A_{\Sigma}$. The first term of the series is the following:

$$
\mathrm{F}=2 \mathrm{Nm}_{\mathrm{v}} \mathrm{c}^{2} \lambda_{1}^{2} \Delta \mathrm{A}_{\Sigma}\left[\Delta\left(\Delta \mathrm{A}_{\Sigma}\right) / \Delta \mathrm{x}\right]
$$

where $\mathrm{N}$ is the number of stable particles (electrons, protons, and neutrons) in the test body, $\Delta \mathrm{A}_{\Sigma}$ is the difference in changes of the summary potential $A_{\Sigma}$ at the location points

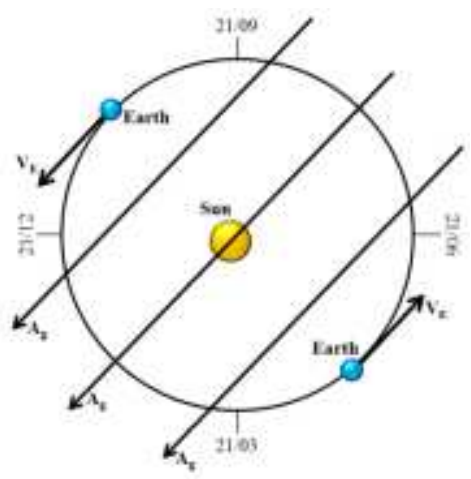

Figure 1. Projection of the cosmological vector potential $A_{g}$ onto a simplified Earth's orbital plane; $V_{E}$ is the Earth's instantaneous velocity vector. 
of a test body and sensor element, $\Delta\left(\Delta \mathrm{A}_{\Sigma}\right) / \Delta \mathrm{x}$ is the gradient in space of the difference potentials $\Delta \mathrm{A}_{\Sigma}$; $\mathrm{x}$ is the length of an arc of a circle for experiments with solenoids, therefore a space coordinate; $2 \mathrm{Nm}_{\mathrm{v}} \mathrm{c}^{2}=33 \mathrm{eV}$; $\lambda_{1}=10^{-6}(\mathrm{Tm})^{-1}$ is the first coefficient of the series.

In [9] the origin of the dark energy of the Universe (about $70 \%$ of total energy) is explained on the basis of the changes of the summary potential of $A_{\Sigma}$ by means of the repulsion of the galaxies which in turn reduces the module of $\mathrm{A}_{\Sigma}$ by means of their own gravitational potentials. Moreover, the mutual repulsion and acceleration of the galaxies is explained in the same work under the theory of byuon, starting from the interaction of two galaxies, each of which containing $10^{10}$ stars, assuming that the mass of each star is of the order of the solar mass $\left(10^{30} \mathrm{~kg}\right)$ and the relative velocity of each galaxy is $\mathrm{v}=100 \mathrm{~km} / \mathrm{s}$ or $\mathrm{v}=1000 \mathrm{~km} / \mathrm{s}$ : the distance $\mathrm{R}_{\mathrm{GG}}$ at which the new force predicted by the theory of byuon will be greater than the gravitational force results as follows: $\mathrm{R}_{\mathrm{GG}} \geqslant 10^{24} \mathrm{~m}$ for $\mathrm{v}=100 \mathrm{~km} / \mathrm{s}$, and $\mathrm{R}_{\mathrm{GG}} \geqslant 10^{26} \mathrm{~m}$ for $\mathrm{v}=1000 \mathrm{~km} / \mathrm{s}$.

As anticipated above, the fundamental research of the global anisotropy of physical space along with the basics of the byuon theory based upon such anisotropy as well as all the relevant bibliography is summarized in[1].

\subsection{New Principle of Universal Propulsion}

The new principle of universal propulsion, i.e. traction force applicable to any object in any surrounding medium, is based upon the role of the physical space as an environment supporting the motion of an object; the physical space is to be meant as a quantum medium filled with "hot" and "cold" dark matter in the framework of the theory of byuon[7-9]. Therefore, it is possible to figure out some new universal class of thrusters able to move any object in any environment, e.g. under the water, on the water and through the air, as well as in the cosmic space, because it will exchange momentum neither with any ordinary substance, nor with any substance ejected from the object itself such as exhaust gases or ionized particles: the momentum exchange will occur with the physical space that in the theory of byuon is not just some mathematical fiction, rather a physical object which density of matter without ordinary substance is around $10^{-29} \mathrm{~g} / \mathrm{cm} 3$ ("cold" dark matter). Close to any usual material bodies, the density of matter is much larger ("warm" dark matter). No need to say that the resulting propulsion, even if based upon a new kind of momentum exchange, must obey Newton's third law of motion.

Any material body can only decrease the summary potential $A_{\Sigma}$ by means of the whole set of its physical potentials[14]: in the physical space a sort of "potential hole" arises with a reduced module of $\mathrm{A}_{\Sigma}$ that will be indicated as the Information Object (IO) of the given body in the physical space. The reality of the IO was investigated for the first time by means of experiments with a physical pendulum, earlier described in[14]; in the course of 600 experiments, the new force was detected and its intensity acting upon the pendulum's load with mass $28 \mathrm{~g}$ was $1.3 \cdot 10^{-3} \mathrm{~N}$ to $4.6 \cdot 10^{-3} \mathrm{~N}(0.13$ gf to $0.47 \mathrm{gf})$; moreover, it was established that the IO of the same body could arise in a time not longer than $0.3 \mathrm{~s}$ and vanished after nor more than $7 \mathrm{~s}$.

\section{Experimental Set-Up and Methods}

In order to avoid most of the systematic errors and to exclude the effect of inertia, i.e. arising from different values of the friction forces in different directions, a prototype of a new concept universal thruster was placed onboard a polycarbonate hemispherical ship model with deviation from sphericity at the level of $0.1 \%$.

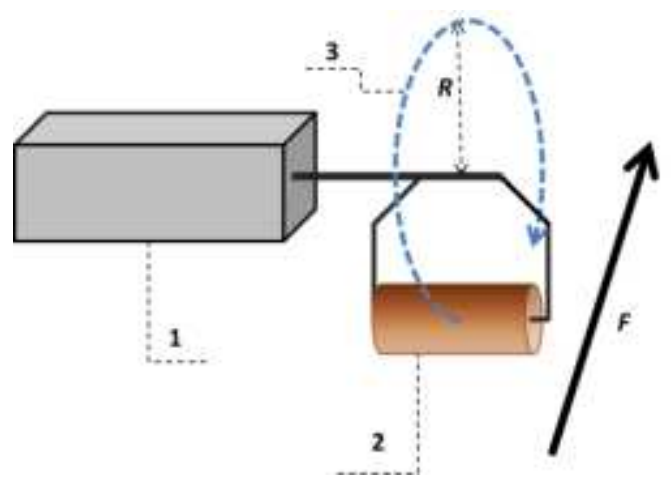

Figure 2. Schematic view of the new thruster. 1 - electromotor (i.e. a step motor); 2 - off-center iron load; 3 - rotational trajectory of the load, periodically resting along or near the vertical; $R$ - revolution radius, not less than $5 \mathrm{~cm} ; \mathrm{F}$ - expected vector of action of the new force, producing cyclic impulses just before each stop of the load, opposite to the direction of motion of the load itself.

Fig. 2 shows the basic elements of the prototype thruster, including a programmable electro-motor, a simple mechanical device nested into the motor's shaft and in turn carrying an off-center cylinder-shape iron load performing revolutions on a vertical plane orthogonal to the motor's shaft at a distance not less than $5 \mathrm{~cm}$ from it. The motor allowed to regulate the load's revolution period, its cyclic stop as well as the load's rest time in the stop position.

Although few experiments were performed with off-center loads having different masses (526 g, $934 \mathrm{~g}$ and $2051 \mathrm{~g}$ ), in the following only the experiments performed with a load with mass equal to $526 \mathrm{~g}$, including supports and bolts, will be described; to make sure that the entire body of the load were more than $5 \mathrm{~cm}$ away from the axis of revolution, its longitudinal axis was fixed at a distance of about $8 \mathrm{~cm}$ from the motor's shaft.

In the following, only the experiments performed with the cyclic stop in the lowest position will be described in order to preserve the symmetry of the load's motion with regards to the vertical direction; nevertheless, it's worth noting that, since in previous experiments with a strain-gauge balance the maximum action of the new force was shown to occur few tenths of degrees $\left(10^{\circ}-30^{\circ}\right)$ before the stop position with direction tangent to the load's motion trajectory, the horizontal component of the impulsive force expected to be 
received with the load stopping in the lowest position will be significantly lower than its absolute value[14].

Three different programmable electro-motors were used in the course of the experimental runs carried out in the period November 2012 to September 2013: a stepper motor model FL110STH201-8004 with nominal torque equal to $2.9 \mathrm{Nm}$ able to perform revolution cycles with periods not smaller than $0.4 \mathrm{~s}$ with angular steps equal to $1.8^{\circ}$, a servo-motor model DSF5.52.2047 with nominal torque equal to $12.9 \mathrm{Nm}$ able to perform revolution cycles with periods as small as $0.115 \mathrm{~s}$, a servo-motor model DSM5.32 with nominal torque equal to $2.4 \mathrm{Nm}$ able to perform revolution cycles with periods not smaller than $0.22 \mathrm{~s}$.

The load's motion in any revolution cycle was programmed by means of the specific motor's controller/encoder, in order to ensure an exact symmetry in the ascending and descending branch of the circular trajectory, regardless of the used motor.

It should be noted that just on the basis of the above mentioned exact symmetry, the proposed prototype of space thruster passes all the criteria on the basis of which any "breakthrough" propulsion ideas proposed in the past and based upon purely mechanical (electro-mechanical) devices have been ruled out[18]: such devices, also called "oscillation thrusters", invariably include motion cycles of few loads (masses) with asymmetric features.

The acceleration in a direction is greater than the acceleration in the opposite direction, so that the reaction force in a direction exceeds the static friction produced by the contact surface and the device moves, while in the opposite direction it doesn't. If no contact surfaces (i.e., friction) are available, such as in the cosmic space, any net motion vanishes and those devices simply cannot work, therefore missing just their claimed use.

The ship model, schematically represented in Fig. 3(a) reproduced from[14], consists of an hemispherical polycarbonate body, with the inner diameter of the sphere equal to $0.9 \mathrm{~m}$, two rigidly connected steel decks to the upper of which is fixed the programmable motor, an autonomous power supply system based on a $12 \mathrm{~V}, 30 \mathrm{Ah}$ battery connected with a transformer/inverter to power themotor with $230 \mathrm{~V}$ alternate current, supplemented with a smaller capacity $24 \mathrm{~V}$ battery to power the encoder when using a servo motor. Any battery is recharged once its voltage decreases by $0.3 \mathrm{~V}$ under its nominal value in order to preserve the optimal performance of the equipment.

Balance weights placed on the same upper steel decks ensure the circular symmetry of the waterline; under the lower deck about $100 \mathrm{~kg}$ of sand are placed for stabilization, so that the total mass is about $120 \mathrm{~kg}$ to $130 \mathrm{~kg}$, depending upon the motor model.

Fig. 3 (b) shows the ship model floating in a water pool having an inner diameter equal to $3.66 \mathrm{~m}$ and a depth between $0.7 \mathrm{~m}$ and $0.9 \mathrm{~m}$.

In Fig. $3 \mathrm{a}$ is also shown the traction force measurement method which includes a very thin cotton thread fixed to the ship model, on one side, passing through a thin metal ring and connected to a set of hanging known weights, onthe other side; the smallest weight unit has been $0.32 \mathrm{~g}$ to $1.0 \mathrm{~g}$; the actual traction force for any set of the off-center load revolution parameters is comprised between a lower value (weight rising during at least $10 \mathrm{~s}$ ) and an upper value (weight falling during at least $10 \mathrm{~s}$ ). Each measurement of the traction is repeated at least five times, until at least $80 \%$ of the measurements fall in the same interval. The resulting traction force is corrected for the observed angle between the thread and the horizontal plane, i.e. dividing by the tangent of such angle, as well as subtracting and adding to the lower and upper values of the traction force, respectively, the upper value of the friction force produced by the water, which value is estimated below. The friction produced by the contact of the cotton thread with the thin metal ring is estimated as negligible after observing that a weight as small as $0.1 \mathrm{~g}$ is able to move the ship model.
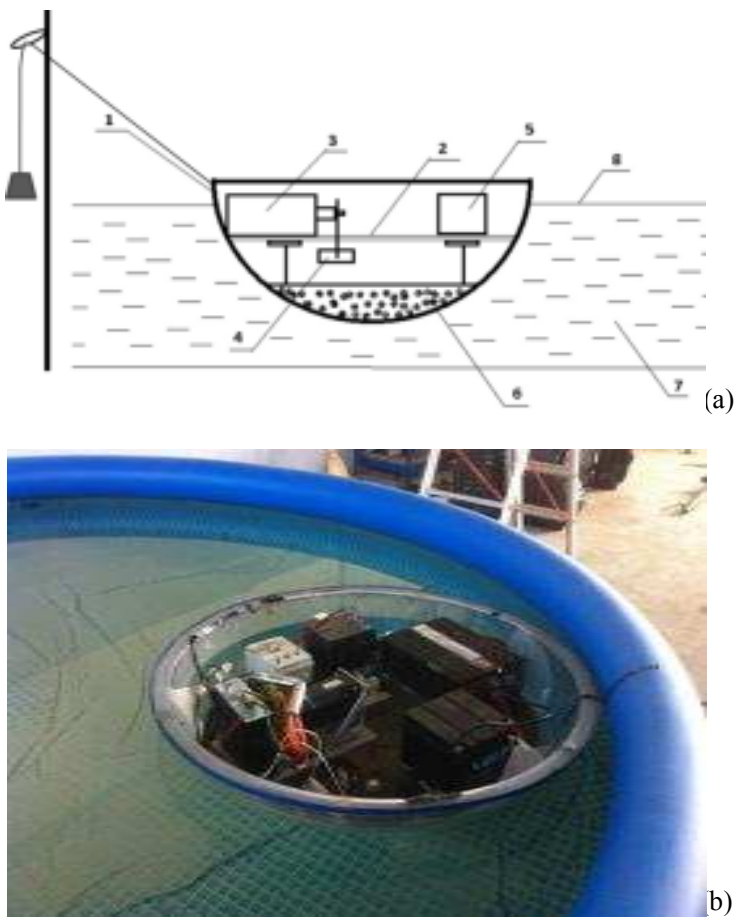

Figure 3.(a)Essential scheme of the model of hemispherical ship with traction generated by the new force of nature. 1 - hemispherical housing; 2 - steel deck; 3 - programmable motor; 4 - rotating load; 5 - power supply system with battery; 6 - additional load (e.g., sand); 7 -water; 8 water surface. The traction force measurement method is also shown on the left. (b) Model of hemispherical ship with new thruster onboard, floating in a $3.66 \mathrm{~m}$ diameter water pool; the $12 \mathrm{~V}$ and $24 \mathrm{~V}$ batteries, the transformer/inverter, a control box, the servo-motor (torque equal to 2.4 $\mathrm{Nm})$ and its encoder are clearly visible.

The upper value of the traction force can be estimated replacing the quantities in (1) with the appropriate numbers; given the mass equal to $526 \mathrm{~g}$ and material, i.e. iron, of the off-center load of revolution we have: $\mathrm{N} \approx 10^{26}$ and, according to[7-9]: $\Delta \mathrm{A}_{\Sigma}=\Delta\left(\Delta \mathrm{A}_{\Sigma}\right), \Delta \mathrm{A}_{\Sigma}=\mathrm{A}_{\mathrm{g}} \cdot \cos _{\text {II I }}^{++} \cdot \mathrm{c} / \mathrm{v}$,

$\cos _{\text {II I }}^{++}=10^{-15}$, where $\mathrm{c}$ is the speed of light, $\mathrm{v}=\Delta \mathrm{x} / \Delta \mathrm{t}=0.02 \mathrm{~m} / \mathrm{s}$ is the extinction velocity of the $\mathrm{IO}$, where $\Delta \mathrm{x}$ is the characteristic size of the body of revolution, i.e. around $2 \mathrm{~cm}$ in the performed experiments, and $\Delta \mathrm{t}$ is the 
extinction time of the IO, on the order of $1 \mathrm{~s}$ as stated in[14]. Substituting into (1), the upper limit of the traction force can therefore be estimated on the order of $1 \mathrm{~N}$.

Having estimated the force and knowing the mass of the ship model (about $120 \mathrm{~kg}$ ), by consequence it takes at least $12 \mathrm{~s}$ for the ship model to reach the speed of $0.1 \mathrm{~m} / \mathrm{s}$ starting from rest: since any measurement of the actual traction force lasts about $10 \mathrm{~s}$, the above value can be assumed as the upper limit for the speed of the ship model in water. Simple computation allows to find that the Reynolds Number $\left(\mathrm{R}_{\mathrm{e}}\right)$ for a sphere with radius around 0.5 $\mathrm{m}$ moving at the speed of $0.1 \mathrm{~m} / \mathrm{s}$ in water is equal to $5 \cdot 10^{4}$, while in water the transition from laminar to turbulent flow occurs for values of $\mathrm{R}_{\mathrm{e}}$ greater than double the above stated value[19].

In laminar water flow, the friction force is proportional to the velocity of the object (the ship model) and is given by the Stokes law[19]: assuming for the sake of simplicity that half of the sphere is completely under the water surface, the viscous friction force turns out to be on the order of $5 \cdot 10^{-4} \mathrm{~N}$, i.e. at least three orders of magnitude less than the upper limit of the estimated traction force. Of course, the viscous force produced by the air is much smaller in comparison with the water and will not be considered further.

To complete the description of the measurement process, just before or after the collection of any numerical data about the traction force, a visual inspection of the actual occurrence of free motion of the ship model over the water pool was performed, in order at least to improve the discrimination of the null force from the non-null force events.

The measured traction force should be regarded as an average value over the whole cycle of the off-center load, since the new force has an impulsive character, i.e. actually it acts only during a small arc of the revolution[14].

The consumed power is computed multiplying the absorbed current, measured by means of a current clamp (amperometer), by the voltage, measured by means of a voltmeter, each measurement repeated after the $12 \mathrm{~V}$ battery (continuous current) and after the transformer/inverter (alternate current), as well as separately for the $24 \mathrm{~V}$ battery where, feeding only the encoder, the power consumption is much smaller.

The average consumed power is shown against the off-center load's period of revolution (Fig. 4a) for the stepper motor and the $12.9 \mathrm{Nm}$ torque servo-motor, with the rest time equal to $0.1 \mathrm{~s}$, as well as against the load's rest time (Fig. 4b) for the stepper motor; the error bars summarize the instrumental errors of the voltmeter and amperometer as well as the standard deviations of the repeated current and voltage measurements; for the stepper motor, the average error of the power data is equal to 2.1 W.
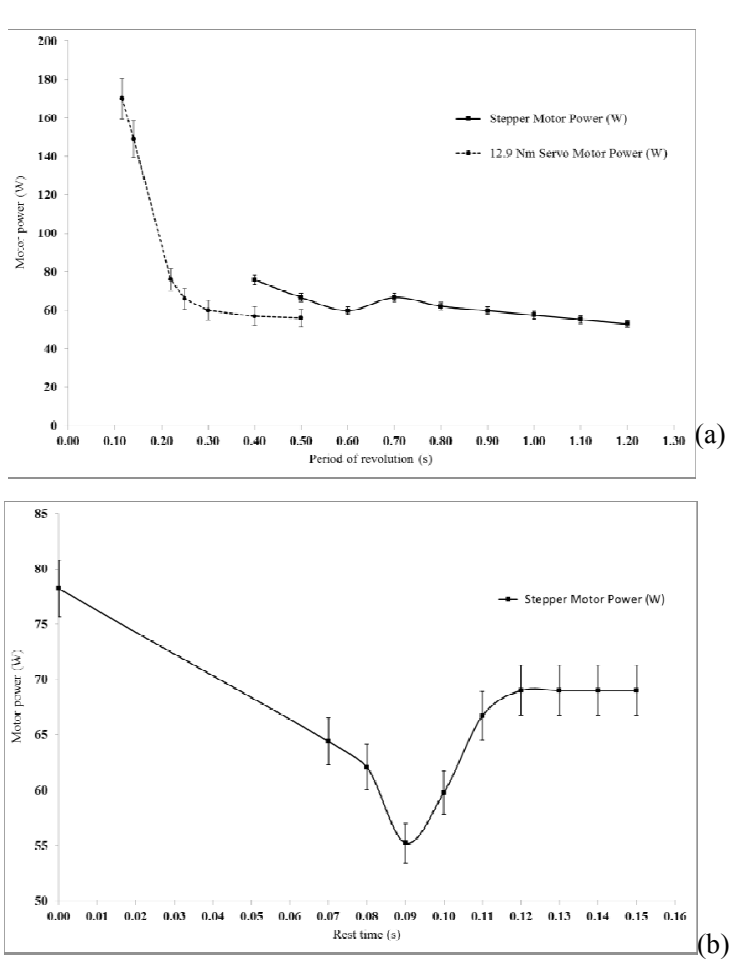

Figure 4. Dependence of the average consumed power (a) on the period of revolution of the off-center load with rest period equal to $0.1 \mathrm{~s}$ and (b) on the rest time of the off-center load with period of revolution equal to $0.6 \mathrm{~s}$.

A good matching between the two curves is apparent in Fig. 4(a), with the servo-motor showing a marginally greater energy efficiency.

About the $2.4 \mathrm{Nm}$ torque servo-motor, it was used for the longest experimental run with constant working parameters, precisely both period of revolution and rest time equal to $0.3 \mathrm{~s}$ : in that case, the average consumed power was measured at the level of $60 \mathrm{~W}$ with similar uncertainty as the stepper motor (around $2 \mathrm{~W}$ to $3 \mathrm{~W}$ ), therefore again matching the measurements shown in Fig. 4(a).

\section{Results and Discussion}

As described in[14], the earlier experiments were carried out in Russia in June, 2012, when the ship model was placed into a $2.5 \mathrm{~m}$ diameter water pool and the load's mass was $718 \mathrm{~g}$ and the same stepper model FL110STH201-8004 was used; those experiments were performed with constant working parameters, precisely period of revolution equal to $0.6 \mathrm{~s}$ and rest time equal to $0.1 \mathrm{~s}$. Shortly, the free motion of the ship model with the new concept thruster was observed and filmed, as well as verified to occur as a linear motion exactly in the expected direction. The maximum measured traction force was a little smaller than $0.1 \mathrm{~N}$ that, with a consumed power less than $100 \mathrm{~W}$, resulted in a specific power on the order of $1000 \mathrm{~W} / \mathrm{N}$.

New practically continuous experiments were performed in Italy in November, 2012, using the same prototype thruster as in the earlier tests, as well as the same spherical ship model, placed in the new water pool (Fig. 3b). Another 
shorter experimental run was carried out between late January and early February, 2013, using the $12.9 \mathrm{Nm}$ servo-motor model DSF5.52.2047 in order to explore shorter revolution periods; finally, a very long experimental run was carried out in the period February to September, 2013, using the $2.4 \mathrm{Nm}$ servo-motor model DSM5.32, mostly with constant working parameters as stated in Section 3. The mass of the off-center load, during both these experiments and the following ones, was $526 \mathrm{~g}$.

Fig. 5 shows the first series of traction force data collected in the period November $5^{\text {th }}$ to $21^{\text {st }}, 2012$, using the stepper motor programmed with a revolution period equal to $0.6 \mathrm{~s}$ and a rest time equal to $0.1 \mathrm{~s}$.

The average traction force over the measurement period takes values around $0.04 \mathrm{~N}$ with few peaks around $0.05 \mathrm{~N}$, along with a significant intra-day and inter-daily variability. While these values substantially confirm the earlier results, as well as allow to rule out any differential friction as a cause of the motion of the ship model since the friction forces are about two orders of magnitude smaller, as computed in Section 3, it's apparent that the straightforward application of (1) produces a traction force more about 7-9 times greater than observed (Section 3).

Recalling from Fig. 4(a) the value of about $60 \mathrm{~W}$ for the average power consumed with the stepper motor programmed with a revolution period equal to $0.6 \mathrm{~s}$ and a rest time equal to $0.1 \mathrm{~s}$, the specific power is on average equal to about $1500 \mathrm{~W} / \mathrm{N}$.

Fig. 6 shows the second and shortest series of traction force data collected in the period January $27^{\text {th }}$ to February $1^{\text {st }}, 2013$, using the $12.9 \mathrm{Nm}$ servo-motor programmed with a revolution period equal to $0.115 \mathrm{~s}$ and a rest time equal to $0.1 \mathrm{~s}$.

The observed values of the traction force are up to three orders of magnitude greater than the computed friction forces, with the largest value of about $0.50 \mathrm{~N}$, which was verified with particular care during many consecutive measurement processes.

Recalling from Fig. 4(a) the value of about $170 \mathrm{~W}$ for the average power consumed with the $12.9 \mathrm{Nm}$ servo-motor programmed with a revolution period equal to $0.115 \mathrm{~s}$ and a rest time equal to $0.1 \mathrm{~s}$, the smallest value of the specific power is equal to about $330 \mathrm{~W} / \mathrm{N}$. It's interesting to note that such value is almost forty times smaller than the corresponding benchmark figure of the most efficient HETs used in the space technology for the propulsion of satellites and spacecrafts, as mentioned in Section 1.

Fig. 7 shows the last and longest series of traction force data collected in the period February $6^{\text {th }}$ to September $15^{\text {th }}$, 2013, using the $2.4 \mathrm{Nm}$ servo-motor programmed with a revolution period equal to $0.3 \mathrm{~s}$, a rest time equal to $0.3 \mathrm{~s}$ and the load's mass equal to $526 \mathrm{~g}$.

Here a striking feature arises: following the apparent fastdecreasing trend observed in Fig. 6, since early February, 2013, a long "shadow zone" with close to about null traction force covers almost exactly six months, before the recovery to normal values during August, 2013.

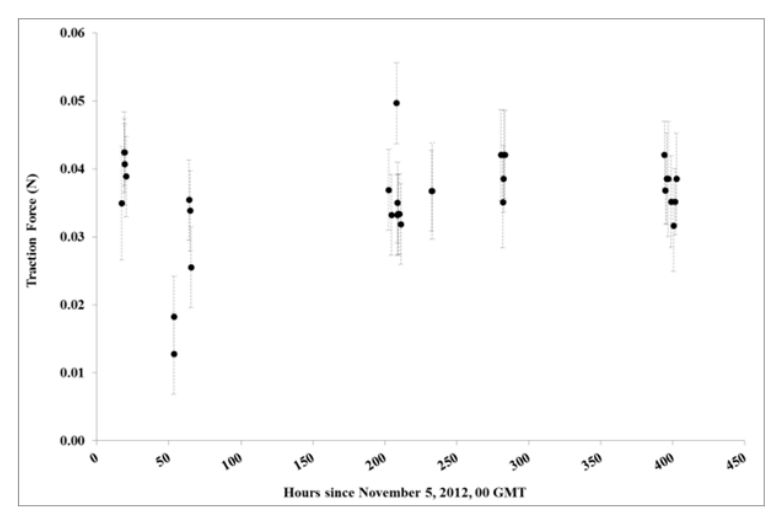

Figure 5. Traction force data series in the period November $5^{\text {th }}$ to $21^{\text {st }}$, 2012. The stepper motor was programmed with a revolution period equal to $0.6 \mathrm{~s}$ and the rest time equal to $0.1 \mathrm{~s}$.

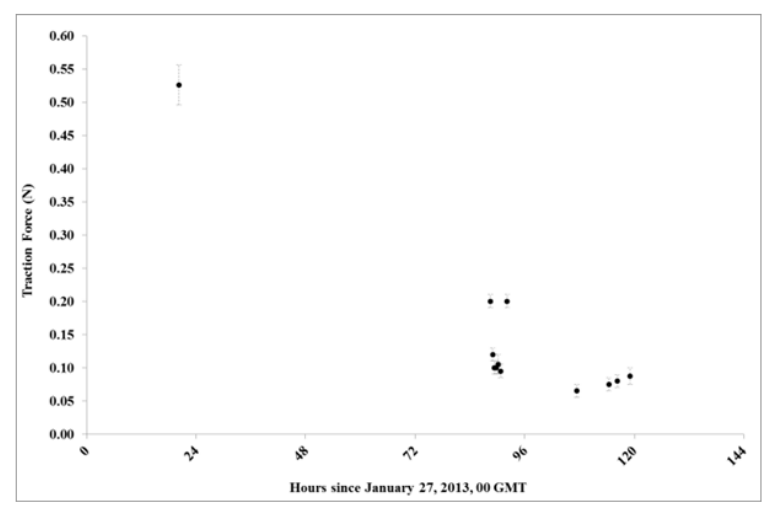

Figure 6. Traction force data series in the period January $27^{\text {th }}$ to February $1^{\text {st }}$, 2013. The $12.9 \mathrm{Nm}$ servo-motor was programmed with a revolutionperiod equal to $0.115 \mathrm{~s}$ and the rest time equal to $0.1 \mathrm{~s}$.

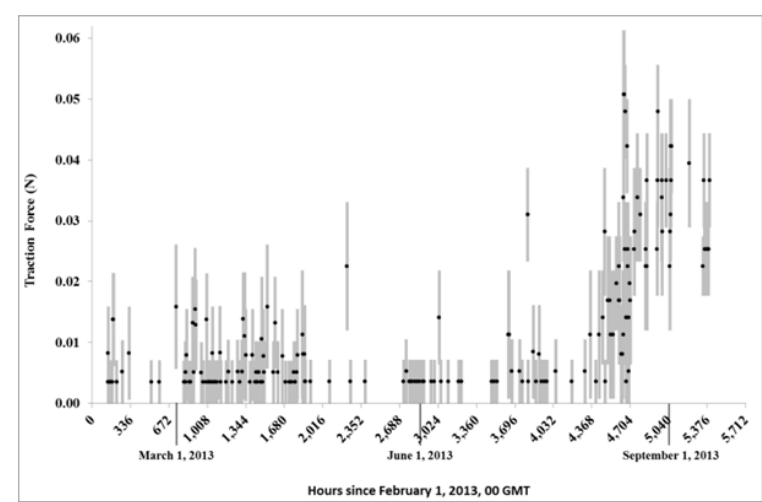

Figure 7. Traction force data series in the period February $6^{\text {th }}$ to September $15^{\text {th }}$, 2013. The $2.4 \mathrm{Nm}$ servo-motor was programmed with a revolution period equal to $0.3 \mathrm{~s}$ and the rest time equal to $0.3 \mathrm{~s}$. The uncertainties of any data point are represented as a light grey shade.

Such fast and significant changes and transitions support the claim that the observed motion of the ship model couldn't be due to any classical known physical effect, given that all the experimental settings and parameters, as well as any known external conditions potentially affecting the motion of the ship model, have not changed.

After its disappearance, the average traction force over the residual measurement period (August $6^{\text {th }}$ to September 
$15^{\text {th }}$ ) takes values around $0.03 \mathrm{~N}$ to $0.04 \mathrm{~N}$ with few peaks around $0.05 \mathrm{~N}$, along with a significant intra-day and inter-daily variability. The presence of few spikes above the null result during the first period suggest that some variability arises even in the course of the shadow zone.

The average consumed power was measured for the only set of working parameters used in this period and is smaller than $60 \mathrm{~W}$, therefore the lowest value of the specific power found in the third experimental run is equal to about $1200 \mathrm{~W} / \mathrm{N}$.

While the sudden transition from the shadow zone (null force) to "normal" value of the thrust represents another convincing support to the claim that the observed motion of the ship model can't be due to any classical known physical effect, as well as another proof of the reality of the new force predicted by the theory of byuon, such shadow zone deserves a deeper explanation.

Fig. 8 shows with a basic scheme the hypothesis of the authors about the origin of the springtime shadow zone. Since Earth's motion of revolution around the Sun (speed around $30 \mathrm{~km} / \mathrm{s}$ ) is prevalent over Earth's motion of rotation around its own axis (speed around $400 \mathrm{~m} / \mathrm{s}$ ), during spring, every point on the Earth's surface moves opposite (anticollinear) to the direction of the cosmological vector potential $A_{g}$ projected onto the Earth's orbital plane, which in turn represents the direction of the global anisotropy of the physical space. The hypothesis is that the IO extinction rate during this period is on average much greater than in fall, when the net velocity of every point on the Earth's surface is collinear to the direction of the cosmological vector potential $\mathrm{A}_{\mathrm{g}}$ projected onto the Earth's orbital plane.

The above described hypothesis implies that the intensity of the repulsive force decreases with increasing IO extinction rate, which is reasonable. The proposed hypothesis needs more extensive verification as well as a comprehensive theoretical framework; at the state of art, anyway, this represents an original contribution of this article.

The origin of the observed intra-daily and inter-daily variability, occurring even in the course of the shadow zones (Fig. 7), should instead be explained on the basis of the Sun's and Earth's magnetic and gravitational potentials [8]; the analysis of such higher frequency variability shall be the subject of further research.

The sensitivity of the new traction force to the period of revolution and to the rest time are assessed on the basis of few measurement sessions closely consecutive in time, carried out during the first and second experimental runs.

Fig. 9 shows the relationship between the traction force and the period of revolution on the basis of the experiments carried out during about 9 hours on November $13^{\text {th }}, 2012$,using the 2.4 Nm stepper motor (a), and during less than 4 hours on January $27^{\text {th }}, 2013$, using the $12.9 \mathrm{Nm}$ servo-motor (b); the rest time was in both cases equal to $0.1 \mathrm{~s}$.

Since the force acts cyclically at any revolution, one might expect it to decrease linearly with increasing period of revolution, and this could be the case with Fig. 9(a); therefore, no reliable conclusion can be drawn on the basisof these data about the time of disappearance of the IO, at least in that given period of the year: on the other hand, it should be remembered that on the basis of the hypothesis proposed above in this Section, the IO extinction rate in fall should be far smaller than in spring.

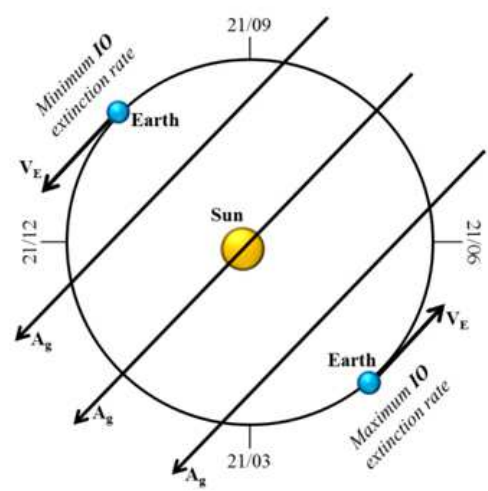

Figure 8. Same as Fig. 1, but showing the hypothesis about the seasonality of the $I O$ extinction rate.

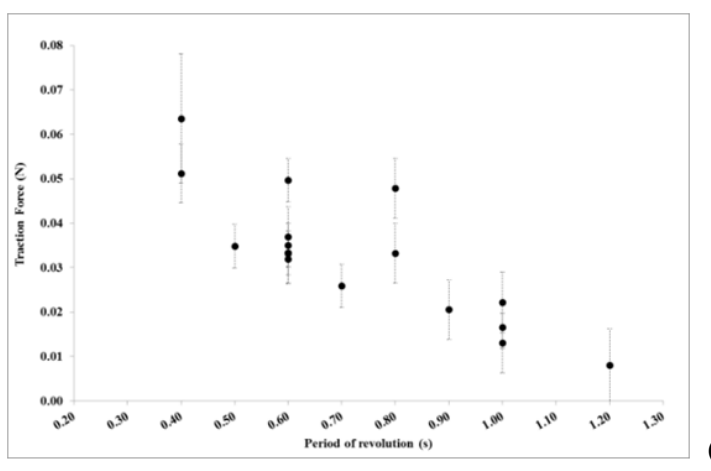

(a)

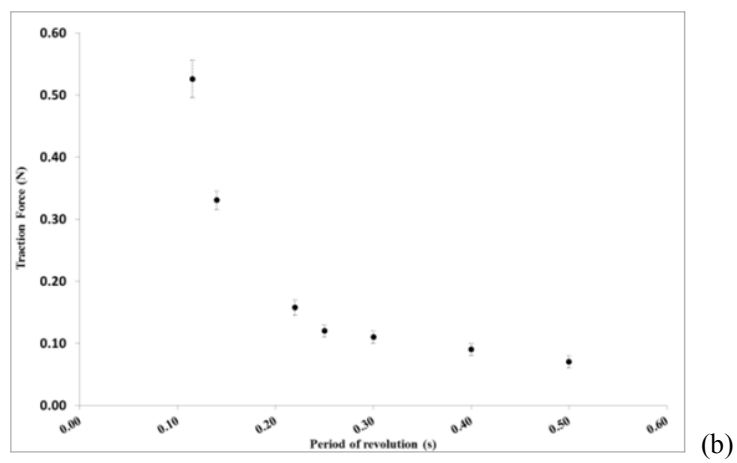

Figure 9. Sensitivity of the traction force to the period of revolution of the off-center load on November 13 $3^{\text {th }}, 2012$, using the $2.4 \mathrm{Nm}$ stepper motor (a), and on January 27 $7^{\text {th }}$ 2013, using the $12.9 \mathrm{Nm}$ servo-motor (b); the rest time was in both cases equal to $0.1 \mathrm{~s}$.

Interestingly, while a linear relationship can be seen in Fig. 9(b) at periods longer than $0.25 \mathrm{~s}$, for shorter periods the traction force appears to grow much faster than linearly with decreasing period, suggesting that the IO extinction rate is far from constant and in particular that such rate decreases in time after the creation of the IO itself, which represents another original contribution of this article.

Fig. 10 shows the relationship between the traction force 
and the rest time of the load in the lowest position of its revolution trajectory on the basis of the experiments carried out during about 22 hours on November $13^{\text {th }}$ and November $14^{\text {th }}, 2012$, using the $2.4 \mathrm{Nm}$ stepper motor; the period of revolution was $0.6 \mathrm{~s}$.

The impulsive force acts cyclically during any revolution, therefore one would expect its average value to decrease linearly with increasing rest time; yet, this is not the case.

At the smallest values, i.e. for rest times equal to $0.08 \mathrm{~s}$ and smaller, the traction force suddenly falls to zero, suggesting the further original conclusion that the creation of the IO takes at least $0.09 \mathrm{~s}$; in the range $0.09 \mathrm{~s}$ to $0.13 \mathrm{~s}$, the force increases, suggesting that the creation of the IO is a gradual process, while for rest times larger than $0.13 \mathrm{~s}$ the force is constant or slowly decreasing, suggesting a sort of "saturation" in correspondence of the rest time equal to $0.13 \mathrm{~s}$.

\section{Conclusions}

A simple experimental device was created and tested with the aim to investigate the existence of a new force of nature predicted by the theory of byuon [1] as well as to harness it in the form of an universal traction force.

In the opinion of the authors, the results of the described experimental runs dissipate any doubt about the existence in nature of a previously unknown interaction that, in contrast to the well-known ones, is not gauge invariant: for its realization the potentials of physical fields must be used, such potentials being considered as non-observable quantities in the usual standard physics.

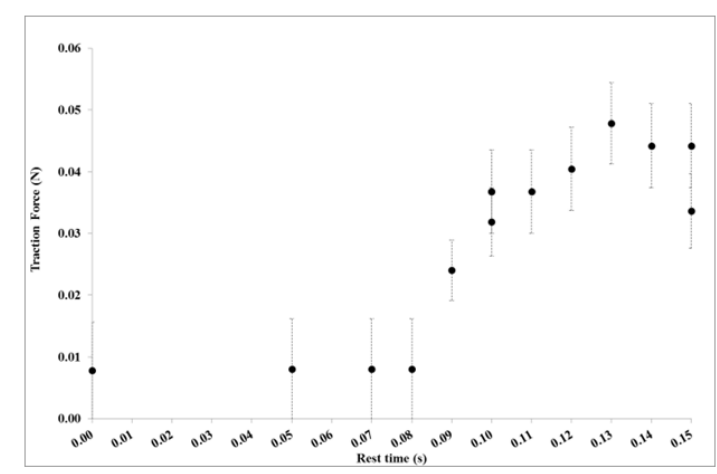

Figure 10. Sensitivity of the traction force to the rest time of the off-center load on November $13^{\text {th }}-14^{\text {th }}$, 2012, using the $2.4 \mathrm{Nm}$ stepper motor. The period of revolution was $0.6 \mathrm{~s}$.

In the existing field theory, potentials are defined only up to an arbitrary constant while only the rate of change of the potentials in space or time makes sense; in the theory of byuon, defining the global anisotropy of the physical space, a new interaction is predicted in connection with the new fundamental vectorial constant $\mathrm{A}_{\mathrm{g}}$ and the field potentials become single-valued since, on the set of byuons, field charge numbers generating the fields themselves are formed, such as, in example, the electric charge of an electron generates an electric field[1,7-9,20].

Beyond the further demonstration of the existence of the new force of nature predicted by the theory of byuon, the other specific, most relevant and original results can be summarized as follows, along with their respective immediate consequences:

- As a new proposed hypothesis, the information object (IO) extinction rate on the Earth is strongly dependent upon its direction of motion in the physical space around the Sun (semi-annual seasonality effect) with regards to the direction of the cosmological vector potential $A_{g}$;

- The IO extinction rate is far from constant and in particular such rate decreases in time after the creation of the IO itself: as a consequence, for periods of revolution of the off-center load in the new thruster shorter than $0.25 \mathrm{~s}$, the traction force grows very quickly with decreasing period;

- The creation of the IO takes at least $0.09 \mathrm{~s}$; in the range $0.09 \mathrm{~s}$ to $0.13 \mathrm{~s}$, the force increases, suggesting that the creation of the IO is a gradual process, while for rest times larger than $0.13 \mathrm{~s}$ the force is constant or slowly decreasing: this evidence suggests a sort of "saturation" in correspondence of the rest time equal to $0.13 \mathrm{~s}$;

- The whole set of results presented in this article and in[14] suggest as well the feasibility of using the new force of nature in the form of traction for some kinds of vehicles, particularly for satellites and spacecrafts: the space flight differs from any other because there's no ordinary material substance or contact surface with which momentum can be exchanged;

- On the basis of the best results obtained and described in Section 4, the experimental device created for the detection and characterization of the predicted traction force appears to be around forty times more energy efficient than the best EP engines, such as the HETs, which are currently deemed to be the most promising for flights to Mars, as mentioned in Section 1.

Future research on this subject shall focus on a more extensive investigation of the sensitivity of the intensity of the traction force to any structural as well as operational parameters recalled in this article, taking into account that the most relevant outcome for practical purposes is the specific power; moreover, new methods involving basic changes to the features of the off-center load in the thruster will be explored to obtain a further substantial increase and stability of the traction force around the year, hopefully overcoming the observed seasonal changes.

\section{Acknowledgments}

The authors gratefully acknowledge G. M. Lanini and G. Tagliaferri for assistance with the preparation of the experimental stands and measurements; C. Dionisio for valuable discussions about space propulsion; M. Pagliaro for the outstanding support and motivation by means of the "SuNEC 2013" Conference; F. Zabini for assistance in the communication style of the manuscript. 


\section{References}

[1] Yu. A. Baurov, "Research of global anisotropy of physical space based on investigation of changes in $\beta$ and $\alpha$-decay rate of radioactive elements , motion of pulsars and anisotropy of cosmic rays," Am. J. Mod. Phys.,vol. 2, pp. 177-184, 2013.

[2] Yu. A. Baurov,E. Yu. Klimenko, and S. I. Novikov,"Experimental observation of space magnetic anisotropy," Phys. Lett. A,vol. 162, pp. 32-34, 1992.

[3] Yu. A. Baurov,"Space magnetic anisotropy and a new interaction in nature," Phys. Lett. A, vol. 181, pp. 283-288, 1993.

[4] Yu. A. Baurov, A. A. Konradov, V. F. Kushniruk, E. A. Kuznetsov, Yu. G. Sobolev, Y. V. Ryabov, et al., "Experimental investigations of changes in beta-decay rate of ${ }^{60} \mathrm{Co}$ and ${ }^{137} \mathrm{Cs}$," Mod. Phys. Lett. A, vol. 16, pp. 2089-2101, 2001.

[5] I. F. Malov, and Yu. A. Baurov,"The distribution of space velocities of radio pulsars," Astron. Reports.vol. 51, pp. 830-835, 2007.

[6] Yu. A. Baurov,"The Anisotropy of Cosmic Rays and the Global Anisotropy of Physical Space," J. Mod. Phys., vol. 03, pp. 1744-8, 2012.

[7] Yu. A. Baurov, On the structure of physical vacuum and a new interaction in Nature (Theory, Experiment and Applications), NY: Nova Science, 2000.

[8] Yu. A. Baurov, Global Anisotropy of Physical Space, Experimental and Theoretical Basis, NY: Nova Science, 2004

[9] Yu. A. Baurov, and I. F. Malov,"On the Nature of Dark Matter and Dark Energy", J. Mod. Phys.,vol. 01, pp. 17-32, 2010.

[10] Yu. A. Baurov, and I. F. Malov, "Variations of Decay Rates of Radio-active Elements and their Connections with Global Anisotropy of Physical Space," Int. J. Pure Appl. Phys., vol. 6, pp. 469-482, 2010. Also at:http://arxiv.org/abs/1001.5383.
[11] M. Dudeck, F. Doveil, N. Arcis, and S. Zurbach,"Plasma propulsion for geostationary satellites for telecommunication and interplanetary missions," IOP Conf. Ser. Mater. Sci. Eng.,vol. 29, 012010, 2012.

[12] A. S. Koroteyev, editor, Manned Mission to Mars. Moscow-Korolev: Russian Academy of Cosmonautics named after K.E. Tsiolkovsky, 2006.

[13] L. Garrigues, and P. Coche, "Electric propulsion: comparisons between different concepts," Plasma Phys. Control Fusion,vol. 53, 124011, 2011.

[14] Yu. A. Baurov, A. Yu. Baurov, A. Yu. J. Baurov, F. Meneguzzo, and A. A. Bugaev,"New Interaction in Nature and its Use in the Form of Traction," Int. J. Pure Appl. Sci. Technol., vol. 13, pp. 40-49, 2012.

[15] Yu. A. Baurov, I. B. Timofeev, V. A. Chernikov, S. F. Chalkin, and A. A. Konradov, "Experimental investigations of the distribution of pulsed-plasma-generator radiation at its various spatial orientation and global anisotropy of space," Phys. Lett. A,vol. 311, pp. 512-523, 2003.

[16] Yu A. Baurov, Yu. G. Sobolev, V. V. Ryabov, and V. F. Kushniruk, "Experimental investigations of changes in the rate of beta decay of radioactive elements," Phys. At. Nucl.,vol.70, pp. 1825-1835, 2007.

[17] P. A. Sturrock, J. B. Buncher, E. Fischbach, D. Javorsek II, J. H. Jenkins, and J. J. Mattes, "Concerning the Phases of the Annual Variations of Nuclear Decay Rates," Astrophys. J.,737:65, 5 pp., 2011.

[18] N. Thomas, NASA Breakthrough Propulsion Physics Project. COMMON ERRORS,http//www.asps.it/errors.htm (last Accessed Oct 21, 2013), 2002.

[19] L. D. Landau, E. M. Lifshitz, Fluid Mechanics, Second Eng. Ed., Oxford: Pergamon Press, 1987.

[20] Yu. A. Baurov, "The anisotropic phenomenon in the $\beta$ decay of radioactive elements and in other processes in nature," Bull. Russ. Acad. Sci. Phys.,vol. 76, pp. 1076-1080, 2012. 